\title{
Electron Microscopy of Sulfur Selenium Alloy with High -K Dielectric Properties.
}

Hector A. Calderon ${ }^{1}$, Francisco C. Robles Hernandez ${ }^{2}$, Pulickel M. Ajayan ${ }^{3}$.

1. Instituto Politécnico Nacional, ESFM Zacatenco, UPALM Ed. \# 9, CDMX, Mexico, 07338.

2. The University of Houston, Mechanical Engineering Technology, Houston, TX, USA, 77204.

3. Department of Materials Science and Nano-engineering, Rice University, Houston, TX, U.S.A, 77030.

Dielectric materials are important passive components in most electrical and electronic circuits used for energy storage, pulsed power, power conditioning, sensing and microelectronics. Conventional dielectrics are either ceramics or polymers. Ceramic dielectrics have a high dielectric constant but are brittle (e.g. BaTiO3, HfO2, etc.), and polymer dielectrics (Polyvinylidene Fluoride, Polyamide or rubber) show a high dielectric strength with flexibility but rather low-K values that limit their application. The full development of this investigation is described elsewhere [1]. It basically deals with a new monolithic, light weight and flexible high K-material produced by combining Selenium (Se) and Sulfur though a co-melting process. The dielectric constant $(\mathrm{K}=74$ at $1 \mathrm{MHz})$ and dielectric strength $(40 \mathrm{kV} / \mathrm{mm})$ are comparable to some values for conventional metal oxides (average $\mathrm{K}=40$ ) and 10 times higher than for polymers $(1<\mathrm{K}<10)$. There is a 13 times increment in dielectric constant as compared to individual components, $\mathrm{S}$ and Se. Here the characterization by electron microscopy is given together with a comparison to the published SSe atomic distributions. The electron microscopy is done in conditions of low dose rates as to preserve the original atomic distribution by using the TEAM05 microscope at $80 \mathrm{kV}$. Exit wave reconstruction (EWR) is used in all cases by using 40 images at different defocus and the software MacTempas ${ }^{\circledR}$ with a dose rate of $10 \mathrm{e}^{-} / \AA^{2}$ s.

Figure 1 shows phase images of different nanoplates after EWR. The different atomic distributions found in nanoplates are clearly shown after a soft Wiener filtering. There is a dominant orientation for the platelets and it is close to [001]. Figure 1 shows in all cases an atomic distribution determined by EWR and by using a rather low dose rate which in most cases ensures the observation of the genuine atomic distribution in the sample. Nevertheless, some images also show areas where bending of the sample is likely since the image tends to deviate from the common orientation for these rather highly sensitive platelets. Figure 2 shows a reference image (Fig. 2a) together with typical results for interatomic distances along parallel (Fig 2b) and perpendicular (2c) directions to the most densely occupied crystalline axis in the image. These measurements are $0.271 \mathrm{~nm}$ and $0.136 \mathrm{~nm}$ in average and coincide within $15 \%$ of the expected interatomic distances for the S3.3Se4.7 phase as published elsewhere [1]. By using this information a simulation is conducted and renders the results shown in Fig. $2 \mathrm{~d}$ which resemble rather closely the experimental results. The simulated image is only one degree off the [001] zone axis of the triclinic structure (space group 14).

\section{References:}

[1] S. Susarla et al., Science Advances, Accepted for publication 2019.

[2] J. Weiss, Zeitschrift fuer Anorganische und Allgemaine Chemie 435 (1977) p113-118.

[3] This research was performed with support of IPN and COFAA-IPN.

[4] Work at the Molecular Foundry was supported by the Office of Science, Office of Basic Energy Sciences, of the U.S. Department of Energy under Contract No. DE-AC02-05CH11231. 

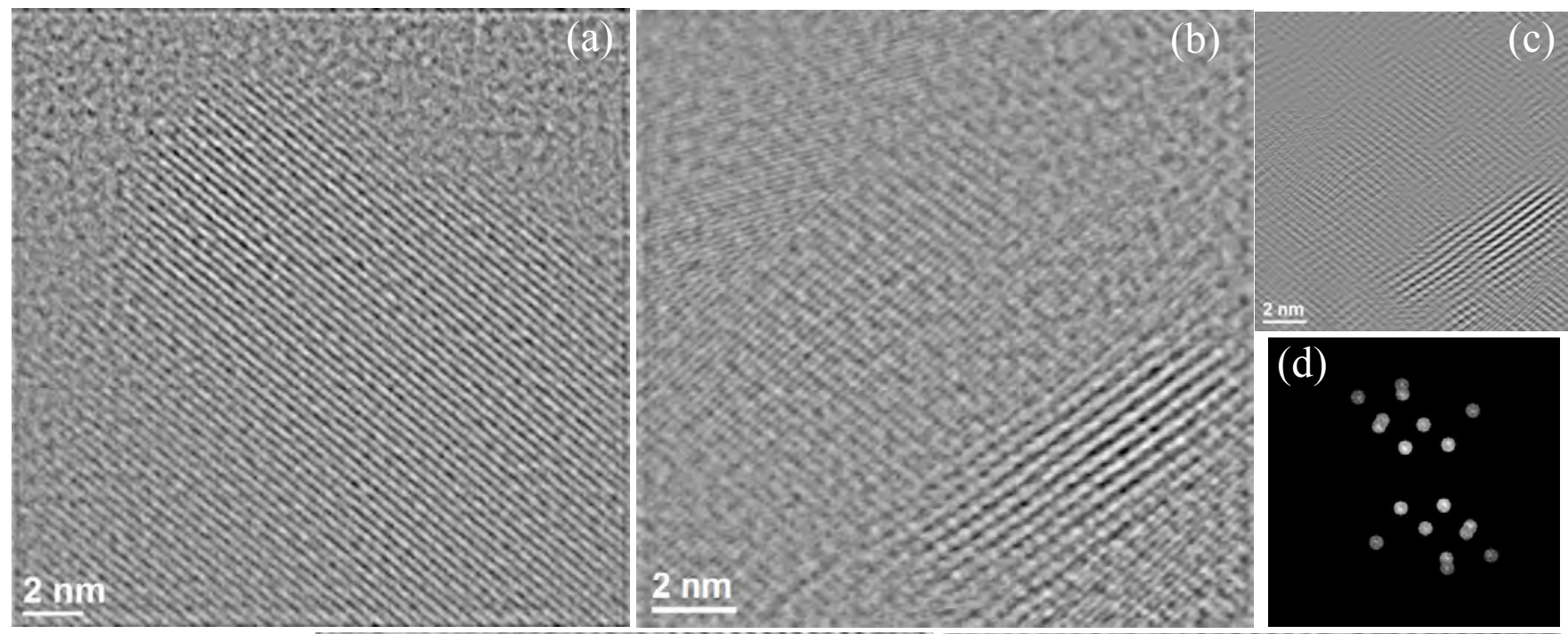

Figure 1. Phase images of $\mathrm{SSe}$ nanoplates after EWR procedure. Wiener filtering has been applied in all cases. (a-b) and (e-f) phase images. (c-d) Inverse Fourier Transform image corresponding FFT.
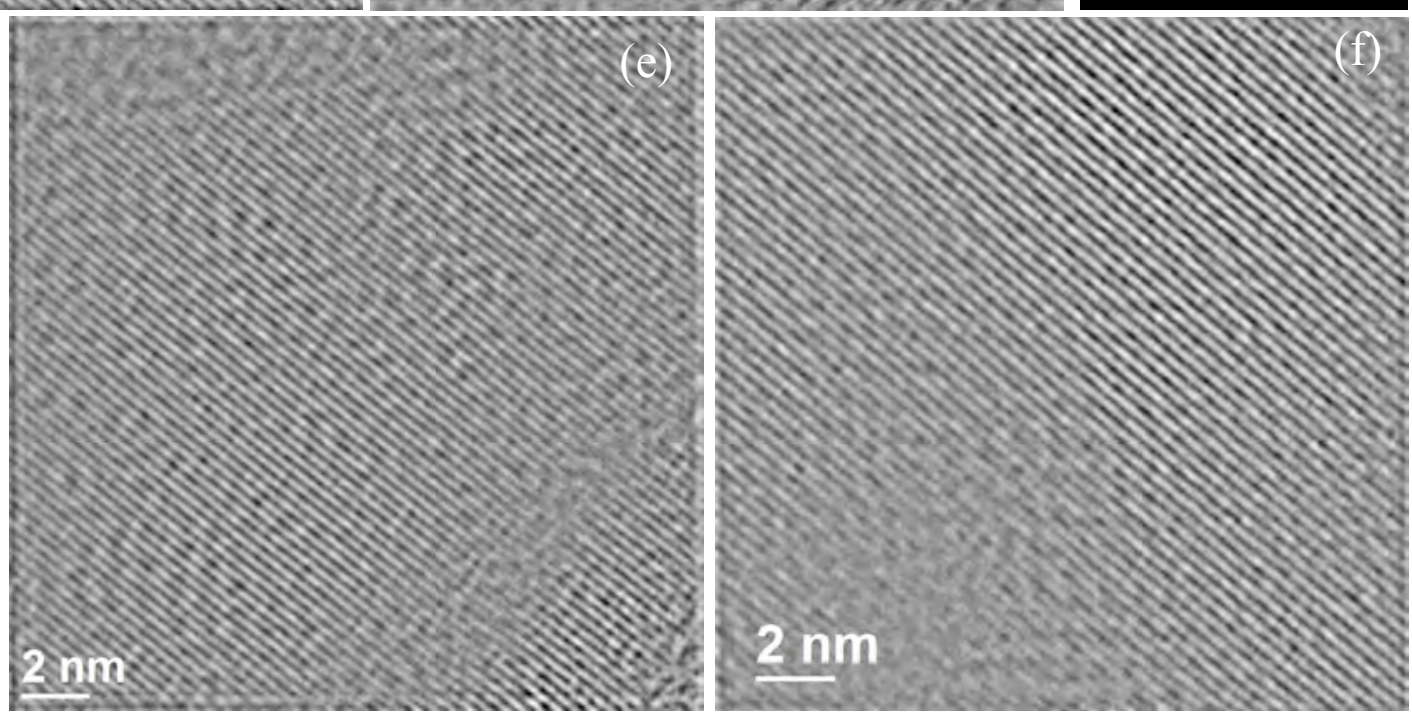

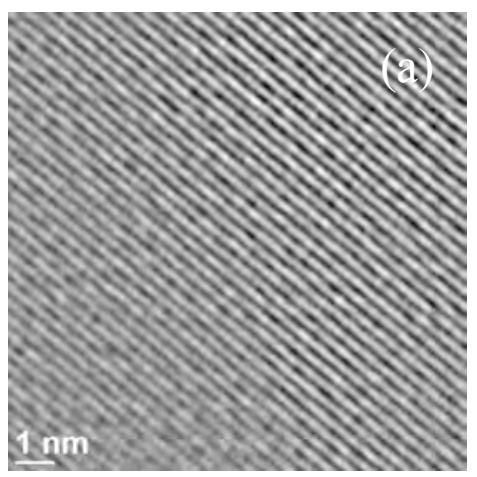

Figure 2. (a) Phase image. (b-c) Characteristic interatomic distances profiles. (d) Simulation of atomic distribution along [001] zone axis for S3.3Se4.7 phase cif file.

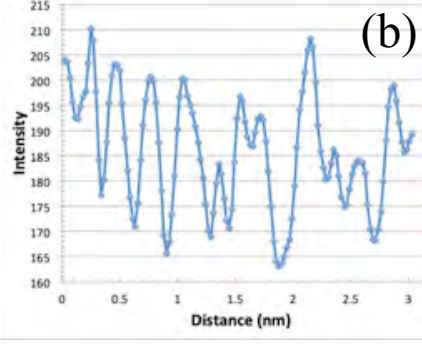

(c)

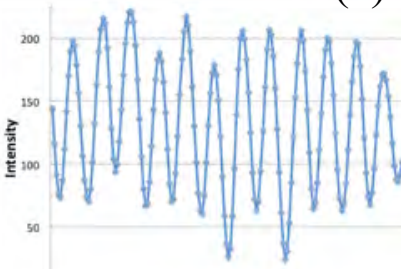

Distance $(\mathrm{nm})$

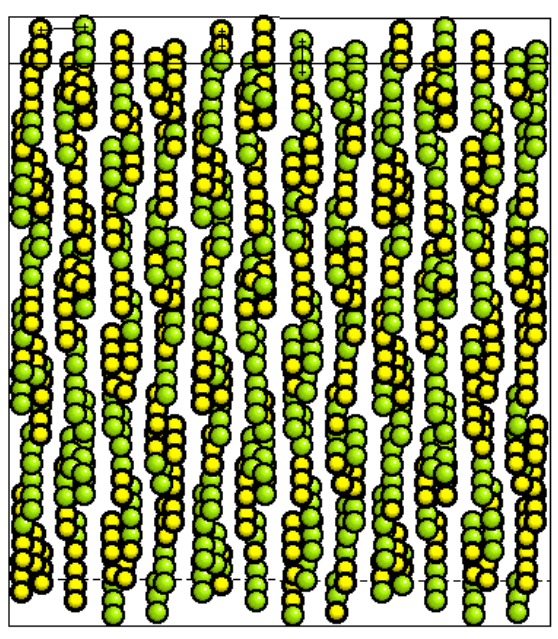

(d) 